\title{
Detection of non-Einsteinian Gravitational Waves using a Pulsar Timing Array
}

\author{
Sydney J. Chamberlin \\ Center for Gravitation and Cosmology \\ Department of Physics \\ University of Wisconsin-Milwaukee \\ Milwaukee, Wisconsin
}

\begin{abstract}
In the next decade gravitational waves may be detected using a pulsar timing array. In an effort to develop optimal detection strategies for stochastic backgrounds of gravitational waves in general metric theories of gravity, we investigate the overlap reduction functions for these theories and discuss their characteristics. We show that sensitivity increases for non-transverse gravitational waves and discuss the physical origin of this effect. We calculate the overlap reduction functions for the current NANOGrav Pulsar Timing Array (PTA) and show that the sensitivity to the vector and longitudinal modes can increase dramatically for pulsar pairs with small angular separations.
\end{abstract}

\section{Introduction}

Advancements in gravitational wave astronomy are making it possible to test Einstein's theory of gravity. The detection of gravitational waves (GWs) could support or rule out modified gravity (non-Einsteinian) theories. Furthermore, the detection of GWs will open a new era of astronomy, making it possible to learn more about astrophysical objects in the universe and the beginnings of the universe itself.

Current GW detection efforts focus primarily on ground-based laser interferometric detectors and the use of pulsar timing arrays. Pulsar timing arrays search for GWs by exploiting the microsecond regularity of pulsar signals. The presence of a GW in the space between the Earth and the pulsar would be manifested by a redshift, $z(t)$, in the pulsar's signal. Radio telescopes are used to determine a quantity known as the timing residual, which is defined as the difference between the actual and expected time of arrival of a pulse:

$$
R(t)=T O A_{\text {actual }}-T O A_{\text {expected }}
$$

The timing residual is directly related to the redshift of the pulse,

$$
R(t)=\int_{0}^{t} d t^{\prime} z\left(t^{\prime}\right)
$$

\footnotetext{
${ }^{0}$ We would like to extend our thanks to the members of the NANOGrav data analysis working group for their comments and support, especially Jim Cordes, Paul Demorest, Justin Ellis, Rick Jenet, Andrea Lommen, Delphine Perrodin, and Joe Romano. We would also like to thank Jolien D. E. Creighton for numerous useful comments and suggestions. This work was funded in part by the Wisconsin Space Grant Consortium and the NSF through CAREER award number 09955929 and PIRE award number 0968126.
} 
which can also be defined geometrically using the techniques discussed by (Detweiler 1979). The metric perturbation due to a GW has the plane-wave expansion

$$
h_{i j}(t, \vec{x})=\sum_{A} \int_{-\infty}^{\infty} d f \int_{S^{2}} d \Omega e^{2 \pi i f t(t-\hat{\Omega} \cdot \vec{x})} h_{A}(f, \hat{\Omega}) \epsilon_{i j}^{A}(\hat{\Omega})
$$

where the sum is over all possible GW polarization modes (plus, cross, breathing, longitudinal, vector $\mathrm{x}$, vector $\mathrm{y}$ ) and $\epsilon_{i j}$ are the polarization tensors given in Table 1 , and for pulsar signals originating in the direction $\hat{p}$ (see Fig. 1), induces the redshift

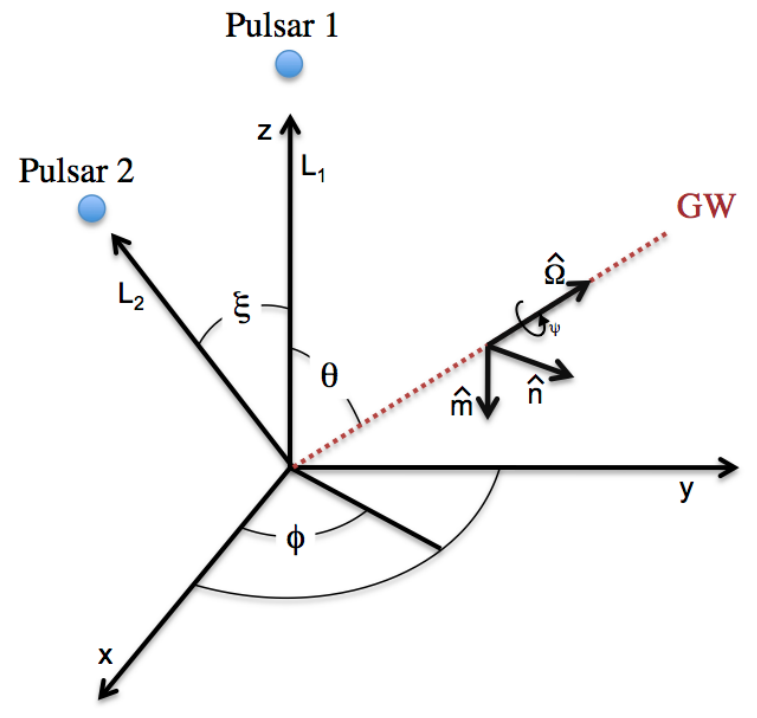

Figure 1: Geometry of the two pulsar-Earth system; $\xi$ is the pulsar separation angle and $L_{1}, L_{2}$ are the distances of the two pulsars from the Earth (origin). Pulsar 1 has been placed on the z-axis for convenience, though the initial choice of basis is arbitrary.

$$
z(t, \hat{\Omega})=\frac{\hat{p}^{i} \hat{p}^{j}}{2(1+\hat{\Omega} \cdot \hat{p})}\left[h_{i j}\left(t_{p}, \hat{\Omega}\right)-h_{i j}\left(t_{e}, \hat{\Omega}\right)\right]
$$

where $t_{p}, t_{e}$ represent the time of pulse emission and the time the pulse is received at the Earth. Writing (3) in the frequency domain, the redshift can be expressed in terms of the antenna patterns $F^{A}(\hat{\Omega})$

$$
\tilde{z}(f, \hat{\Omega})=\left(e^{-2 \pi i f L(1+\hat{\Omega} \cdot \hat{p})}-1\right) \sum_{A} h_{A}(f, \hat{\Omega}) F^{A}(\hat{\Omega}) .
$$

For a stochastic background of GWs, the signal appears in data as correlated noise between measurements from different detectors. The output of the $i^{\text {th }}$ detector is of the form

$$
s_{i}(t)=z_{i}(t)+n_{i}(t)
$$




\begin{tabular}{cc}
\hline \hline$\epsilon_{a b}^{+}=\hat{m} \otimes \hat{m}-\hat{n} \otimes \hat{n}$ & $\epsilon_{a b}^{\times}=\hat{m} \otimes \hat{n}+\hat{n} \otimes \hat{m}$ \\
$\epsilon_{a b}^{b}=\hat{m} \otimes \hat{m}+\hat{n} \otimes \hat{n}$ & $\epsilon_{a b}^{l}=\hat{\Omega} \otimes \hat{\Omega}$ \\
$\epsilon_{a b}^{x}=\hat{m} \otimes \hat{\Omega}+\hat{\Omega} \otimes \hat{m}$ & $\epsilon_{a b}^{y}=\hat{n} \otimes \hat{\Omega}+\hat{\Omega} \otimes \hat{n}$ \\
\hline \hline
\end{tabular}

Table 1: Polarization tensors

where $z_{i}(t)$ corresponds to the unknown GW signal and $n_{i}(t)$ to noise (assumed in this case to be stationary and Gaussian). For coincident, co-aligned detectors, the cross-correlation statistic is defined as

$$
S=\int_{-T / 2}^{T / 2} d t \int_{-T / 2}^{T / 2} d t^{\prime} s_{1}(t) s_{2}\left(t^{\prime}\right) \delta\left(t-t^{\prime}\right) .
$$

In general, detectors are neither coincident nor co-aligned and it is necessary to generalize the delta function in (6). This generalization appears in the statistic as

$$
S=\int_{-T / 2}^{T / 2} d t \int_{-T / 2}^{T / 2} d t^{\prime} s_{1}(t) s_{2}\left(t^{\prime}\right) Q\left(t-t^{\prime}\right)
$$

where $Q\left(t-t^{\prime}\right)$ is the filter function. The optimal detection statistic is found by optimizing the filter function; this method is known as matched filtering and is the primary strategy for GW signal detection. To do the matched filtering, the expected signal-to-noise ratio

$$
S N R=\frac{\langle S\rangle^{2}}{\sqrt{\left\langle S^{2}\right\rangle-\langle S\rangle^{2}}}
$$

is maximized to find the optimal filter function. In the frequency domain, this appears as

$$
\tilde{Q}(f)=\chi \frac{\Omega_{G W}(|f|) \Gamma(|f|)}{|f|^{3} P_{1}(f) P_{2}(f)}
$$

where $\Omega_{G W}(|f|)$ is the stochastic GW spectrum, $P_{1}(f)$ and $P_{2}(f)$ are power spectra, $\chi$ is a normalization constant and $\Gamma(|f|)$ is the overlap reduction function, which characterizes the loss of sensitivity due to detectors being non-coincident and non-aligned.

The overlap reduction function is defined as

$$
\Gamma(|f|)=\beta \sum_{A} \int_{S^{2}} d \Omega\left(e^{\left\{2 \pi i f L_{1}\left(1+\hat{\Omega} \cdot \hat{p}_{1}\right)\right\}}-1\right)\left(e^{\left\{-2 \pi i f L_{2}\left(1+\hat{\Omega} \cdot \hat{p}_{2}\right)\right\}}-1\right) F_{1}^{A}(\hat{\Omega}) F_{2}^{A}(\hat{\Omega})
$$

where the sum is over all possible GW polarizations (Lee et al. 2008) and the exponential terms (or so-called pulsar terms) describe the metric perturbation at each pulsar. In the regime of alternative gravity theories, each term in the sum may be explicitly computed, i.e.

$$
\Gamma(|f|)=\Gamma_{+}(|f|)+\Gamma_{\times}(|f|)+\Gamma_{b}(|f|)+\Gamma_{l}(|f|)+\Gamma_{x}(|f|)+\Gamma_{y}(|f|),
$$

and it is advantageous to consider each term in (10) separately since various gravity theories require different polarizations (Will 1993; Nishizawa et al. 2009; Lobo 2008; Alves et al. 2009; Capozziello and Francaviglia 2008; De Felice and Tsujikawa 2010; Brunetti et al. 1999; Clifton et al. 2011; Sagi 2010; Clifton et al. 2010; Skordis 2009; Milgrom 2009). It is convenient to note at this point that the first three terms listed in (10) correspond to transverse wave propagation (the first two being the modes of General Relativity), while the latter three correspond to non-transverse wave propagation. 


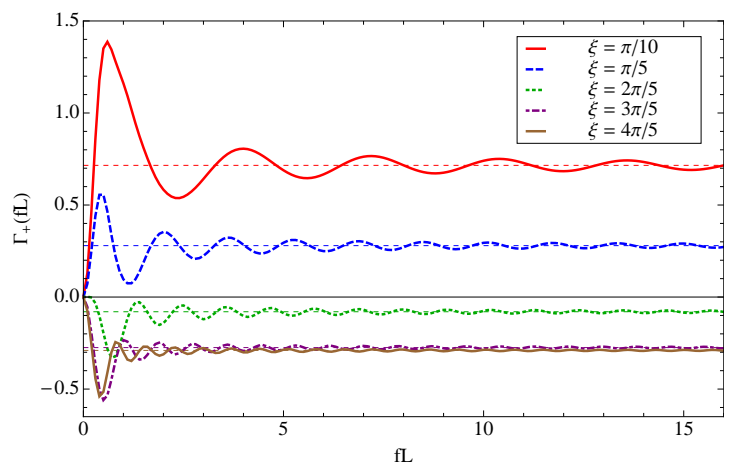

(a)

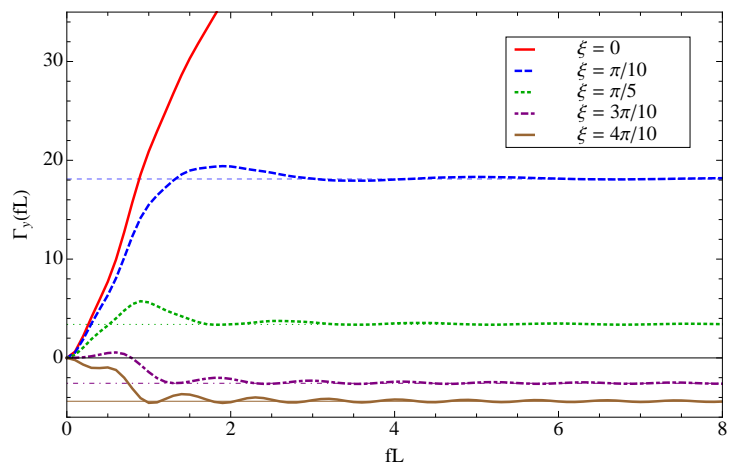

(c)

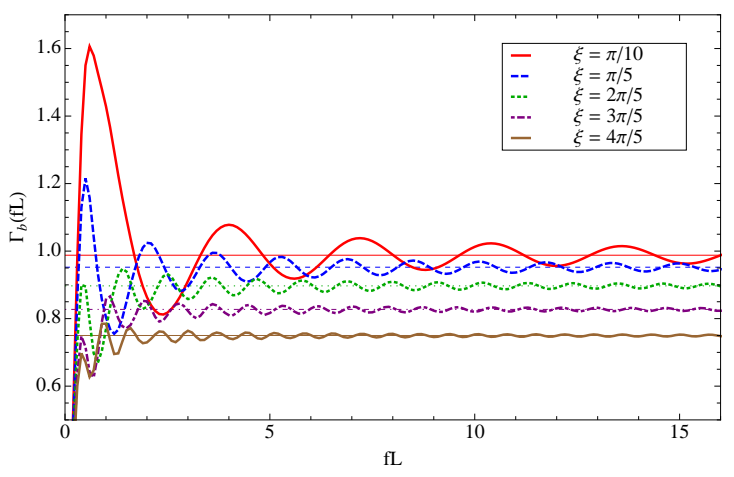

(b)

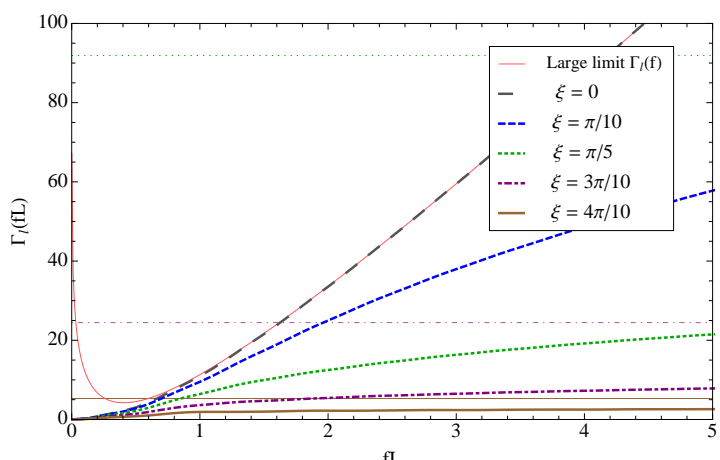

(d)

Figure 2: $\Gamma(f L)$ for the various polarization modes: plus (a), breathing (b), shear (c) and longitudinal (d). In the latter two modes, smaller pulsar separation angles correspond to retained frequency dependence in $\Gamma(f L)$. Nearly all the non-transverse curves eventually converge, but at rather high values of $\Gamma(f L)$ relative to the transverse modes, indicating increased sensitivity to GWs with these polarizations.

\section{Overlap reduction functions}

The overlap reduction function has a closed analytic form for transverse GWs. The overlap reduction function for the plus mode has been calculated by (Anholm et al. 2009) and is given by

$$
\Gamma_{+}(\xi)=3\left[\frac{1}{3}+\frac{1-\cos \xi}{2}\left[\log \left(\frac{1-\cos \xi}{2}\right)-\frac{1}{6}\right]\right],
$$

where $\xi$ is the angular separation of the pulsars. For the breathing mode, a closed form is given by (Lee et al. 2008):

$$
\Gamma_{b}(\xi)=\frac{3}{4 \pi} \frac{\pi}{3}(3+\cos \xi) .
$$

For the case of non-transverse GWs, the overlap reduction function cannot be integrated analytically and must be treated numerically. 
In general relativity the pulsar term is typically excluded from the integral (10) without any loss of optimality (Anholm et al. 2009); see Fig. 2a. The same is not true for scalar and vector GW modes. For non-transverse GW polarization modes, frequency must be retained in the overlap reduction function for pulsars that are nearly co-aligned in order to maintain optimality (Fig. 2). Furthermore, larger values of the non-transverse overlap reduction functions (Figs. 2) indicate increased sensitivity to these modes, in agreement with the results of (Lee et al. 2008; Alves and Tinto 2011). This behavior is absent for transverse GW polarization modes.

The effect on non-transverse GW polarization modes can be understood by studying the induced frequency changes for GWs of different polarizations. Consider the spacetime metric due to a longitudinal mode GW perturbation:

$$
g_{a b}=\eta_{a b}+h_{a b}(t-z) .
$$

It can be shown using (12) that the redshift for this perturbation is

$$
\tilde{z}_{l}(f, \hat{z})=\frac{\cos ^{2} \theta}{2(1+\cos \theta)}\left(e^{-2 \pi i f L(1+\cos \theta)}-1\right) h_{l} .
$$

The analogous redshift for a plus mode GW perturbation is

$$
\tilde{z}_{+}(f, \hat{z})=\frac{-\sin ^{2} \theta}{2(1+\cos \theta)}\left(e^{-2 \pi i f L(1+\cos \theta)}-1\right) h_{+} .
$$

In the region where $\theta \rightarrow \pi$, (13) and (14) appear to become singular, though a simple series expansion shows that only the longitudinal mode retains any unusual behavior in this sky region: Let $\theta=\pi-\delta$, where $\delta$ is a real parameter satisfying $\delta \ll 1$. One may easily conclude that

$$
\tilde{z}_{l}(f, \hat{z}) \sim \pi i f L\left(1-\delta^{2}\right) h_{l}
$$

for the longitudinal case, while

$$
\tilde{z}_{+}(f, \hat{z}) \sim \pi i f L \delta^{2} h_{+}
$$

for the plus mode. In the limit as $\delta \rightarrow 0, \tilde{z}_{+}$vanishes while $\tilde{z}_{l}$ approaches a constant proportional to $f L$. In the time domain, the longitudinal mode redshift for $\theta \approx \pi$ goes as

$$
z_{l}(t, \hat{z}) \propto L \dot{h}
$$

The right hand side of (17) may be identified as a velocity.

For co-aligned pulsars in the problematic sky region $(\theta \approx \pi), \Gamma_{l}(f)$ is proportional to the square of the redshift,

$$
\Gamma_{l}(f) \propto-2 \pi \int_{-1}^{1} d(\cos \theta)\left|\left(e^{-2 \pi i f L(1+\cos \theta)}-1\right)\right|^{2} \frac{\cos ^{4} \theta}{4(1+\cos \theta)^{2}}
$$

which may be evaluated analytically. In the limit of large $f L$,

$$
\begin{aligned}
\Gamma_{l}(f) & =\pi\left\{37 / 6-4 \gamma-1 /\left(\pi(f L)^{2}\right)+4 \operatorname{Ci}(4 \pi f L)\right. \\
& -4 \log (4 \pi f L)+2 \pi f L \operatorname{Si}(4 \pi f L)\}(f L \gg 1) \\
& \sim(37 / 6-4 \gamma) \pi{ }_{5} 4 \pi \log (4 \pi f L)+\pi^{3} f L
\end{aligned}
$$


so that $\Gamma_{l}(f)$ goes like $f L$ up to some large frequency ${ }^{1}$. This large limit $\Gamma_{l}(f)$ is plotted with the overlap reduction functions in Fig. $2 d$ and, with the exception of the singular behavior near the origin, fits the $\Gamma_{l}(f)$ curve for co-aligned pulsars $(\xi=0)$. As $\xi$ increases, correction terms in (19) restrict $f$ dependence of $\Gamma(f)$ until it is approximately constant as a function of frequency.

An identical analysis for the shear GW modes produces similar results. Given a metric perturbation of the same form as (12), the redshift for the vector-y mode goes as

$$
\tilde{z}_{y}(f, \hat{z})=\frac{-\cos \theta \sin \theta}{(1+\cos \theta)}\left(e^{-2 \pi i f L(1+\cos \theta)}-1\right) h_{y} .
$$

Doing the expansion about $\delta$,

$$
\tilde{z}_{y}(f, \hat{z}) \sim-2 \pi i f L \delta\left(1-\frac{\delta^{2}}{2}\right) h_{y} .
$$

As in (19), $\tilde{z}(f, \hat{z})$ retains dependence on $f L$ near $\theta=\pi$, but the growth in $f L$ is constrained by a factor of $\delta$ (see Fig. 2c).

The same behavior is not present in other sky locations. If the GW propagates perpendicular to the earth-pulsar line $(\theta=\pi / 2+\delta)$, then up to second order in $\delta$ the redshifts

$$
\begin{aligned}
\tilde{z}_{l} & =\left(e^{-2 \pi i f L(1-\delta)}-1\right) \frac{-\delta^{2}}{2(1-\delta)} \text { (longitudinal) } \\
\tilde{z}_{y} & =\left(e^{-2 \pi i f L(1-\delta)}-1\right) \frac{\delta}{(1-\delta)}(\text { shear }) \\
\tilde{z}_{+} & =\left(e^{-2 \pi i f L(1-\delta)}-1\right) \frac{-1}{2(1-\delta)} \text { (plus) }
\end{aligned}
$$

are obtained. Even while $\delta$ is small, the exponential cannot be fully expanded. Oscillatory behavior dominates the redshift in this sky location. Physically, this is due to the fact that the pulse "sees" different phases of the GW and the contribution to the redshift varies based on this phase.

In the regime where $\theta \approx \pi$, the pulse is effectively in the long wavelength limit in which the redshift can increase monotonically up to some limiting $f$ at which point the Taylor series expansion of the pulsar term (15) is no longer valid. Given a large initial "kick", the velocity term $L \dot{h}$ becomes quite large. This is what is responsible for the much larger values of the longitudinal and shear mode overlap reduction functions compared to those of the transverse modes.

\section{Overlap reduction functions for the NANOGrav pulsars}

The NANOGrav PTA consists of 24 pulsars. Australia Telescope National Facility data

\footnotetext{
${ }^{1}$ Here $\gamma$ is Euler's constant.
} 


\begin{tabular}{cc|cc}
\hline \hline PSRJ & Distance (kpc) & PSRJ & Distance (kpc) \\
\hline J0030+0451 & 0.23 & $\mathrm{~J} 1853+1303$ & 1.60 \\
$\mathrm{~J} 0218+4232$ & 5.85 & $\mathrm{~J} 1857+0943$ & 0.70 \\
$\mathrm{~J} 0613-0200$ & 2.19 & $\mathrm{~J} 1903+0327$ & 6.45 \\
$\mathrm{~J} 1012+5307$ & 0.52 & $\mathrm{~J} 1909-3744$ & 0.55 \\
$\mathrm{~J} 1024-0719$ & 0.35 & $\mathrm{~J} 1910+1256$ & 1.95 \\
$\mathrm{~J} 1455-3330$ & 0.74 & $\mathrm{~J} 1918-0642$ & 1.40 \\
$\mathrm{~J} 1600-3053$ & 2.67 & $\mathrm{~J} 1939+2134$ & 3.58 \\
$\mathrm{~J} 1640+2224$ & 1.19 & $\mathrm{~J} 1944+0907$ & 1.28 \\
$\mathrm{~J} 1643-1224$ & 4.86 & $\mathrm{~J} 1955+2908$ & 5.39 \\
$\mathrm{~J} 1713+0747$ & 0.89 & $\mathrm{~J} 2010-1323$ & 1.29 \\
$\mathrm{~J} 1738+0333$ & 1.97 & $\mathrm{~J} 2145-0750$ & 0.50 \\
$\mathrm{~J} 1744-1134$ & 0.17 & $\mathrm{~J} 2317+1439$ & 1.89 \\
\hline \hline
\end{tabular}

Table 2: NANOGrav Pulsar Data

for these pulsars is given in Table 2 (Manchester et al. 2005). Using numerical techniques, the overlap reduction function for each pulsar pair was computed. Results are given in Fig. $3 \mathrm{a}-\mathrm{d}$ and show that the calculated values of the $\Gamma(f)$ are consistent with analytical results discussed in previous sections of this document. for the non-transverse modes for frequencies up to $\sim 10^{-9} \mathrm{~Hz}$.

Pulsar pairs with the smallest $\left(\xi<12^{\circ}\right)$ separation angles (starred curves in Fig. 3 $\mathrm{b}, \mathrm{d})$ are characterized by large values of the overlap reduction function and monotonic growth up to some limiting frequency. Pulsar pairs with larger $\left(\xi>12^{\circ}\right)$ separation angles (un-starred curves in Fig. 3 b, d and all curves in Fig. 3) do not display monotonic growth up to a limiting frequency, but still obtain much larger values than those of the plus and cross modes. Fig. 3 shows that sensitivity is greater for scalar and vector modes than tensor modes, and increases rapidly for pulsars that are nearly co-aligned in the sky. Theoretical sensitivity estimates using (19) support this result. In these plots, increased variation in curve amplitudes appears due to the fact that the pulsars are not equidistant from the Earth.

Over the entire range of frequencies relevant to pulsar timing experiments (up to $\sim 10^{-7}$ ), the overlap reduction functions are roughly constant. In practice, some optimality is lost due to the fact that pulsar distances are known at best to only $\sim 10 \%$ (Cordes and Lazio 2002).

\section{Discussion}

Direct detection of GWs is possible in the next decade using a pulsar timing array. This detection will provide a mechanism for testing various metric theories of gravity. To develop robust detection strategies, we have analyzed overlap reduction functions for all possible GW polarization modes. For non-transverse GWs, there exists a region of the sky for which the pulse is in the long wavelength limit, leading to a frequency dependent 


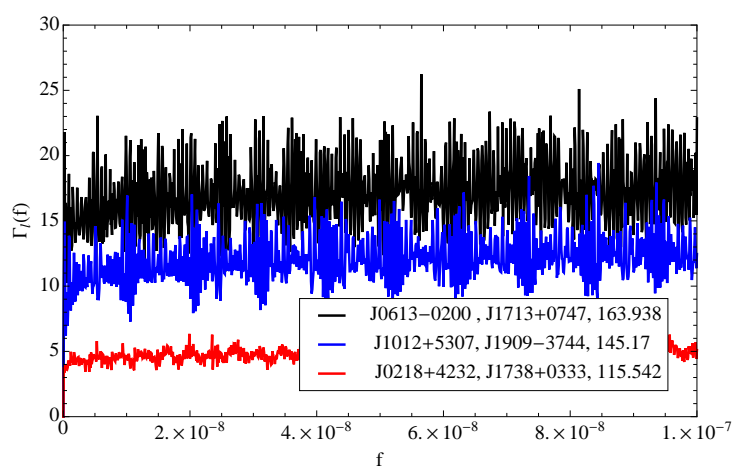

(a)

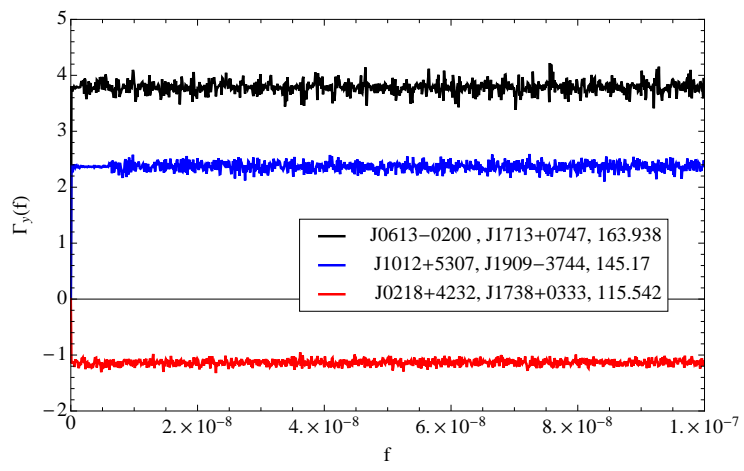

(c)

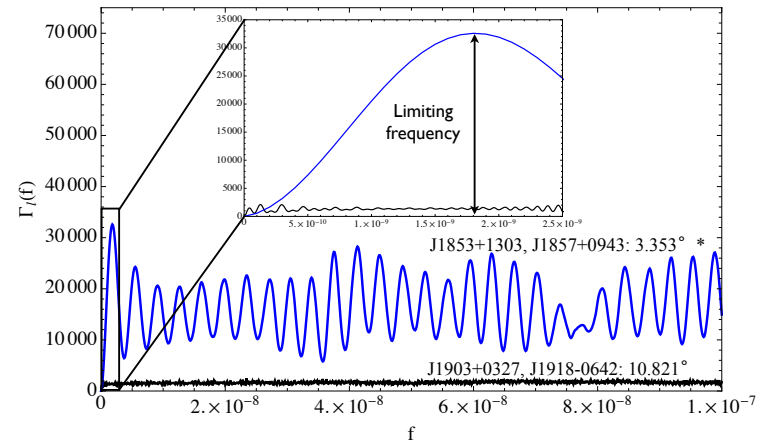

(b)

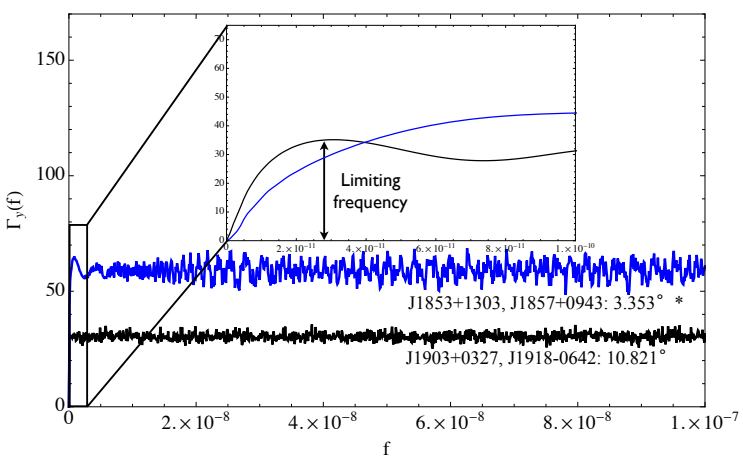

(d)

Figure 3: $\Gamma(f)$ for some of the NANOGrav pulsar pairs. Pulsar pairs, along with their angular separation in degrees, are given in the legend. As $f$ increases, $\Gamma(f)$ approaches a constant value. The asterisk indicates the NANOGrav pulsar pair with the smallest angular separation ( 3.35 degrees). Note the larger values of the $\Gamma(f)$ s for this pair. 
overlap reduction function. Large values of $\Gamma(f)$ for these non-transverse modes indicates increased sensitivity to scalar-longitudinal and vector GWs. A study of current data from the NANOGrav collaboration indicates that pulsar timing measurements are in the optimal regime for most pulsars.

\section{References}

S. Detweiler, Astrophys. J. 234, 1100 (1979).

K. J. Lee, F. A. Jenet, and R. H. Price, Astrophys. J. 685, 1304 (2008), URL http://stacks.iop.org/0004-637X/685/i=2/a=1304.

C. M. Will, Theory and Experiment in Gravitational Physics (Cambridge University Press, 1993).

A. Nishizawa, A. Taruya, K. Hayama, S. Kawamura, and M.-a. Sakagami, Phys. Rev. D 79, 082002 (2009), URL http://link.aps.org/doi/10.1103/PhysRevD.79.082002.

F. S. N. Lobo, ArXiv e-prints (2008), 0807.1640.

M. E. S. Alves, O. D. Miranda, and J. C. N. de Araujo, Phys. Lett. B 679, 401 (2009), 0908.0861.

S. Capozziello and M. Francaviglia, Gen. Rel. Grav. 40, 357 (2008), 0706.1146.

A. De Felice and S. Tsujikawa, Living Rev. Rel. 13, 3 (2010), 1002.4928.

M. Brunetti, E. Coccia, V. Fafone, and F. Fucito, Phys. Rev. D 59, 044027 (1999), URL http://www . citebase.org/abstract?id=oai : arXiv.org: gr-qc/9805056.

T. Clifton, P. G. Ferreira, A. Padilla, and C. Skordis, ArXiv e-prints (2011), 1106. 2476.

E. Sagi, Phys. Rev. D 81, 064031 (2010), 1001.1555.

T. Clifton, M. Banados, and C. Skordis, Class. Quant. Grav. 27, 235020 (2010), 1006.5619 .

C. Skordis, Class. Quant. Grav. 26, 143001 (2009), 0903.3602.

M. Milgrom, Phys. Rev. D 80, 123536 (2009), 0912.0790.

M. Anholm, S. Ballmer, J. D. E. Creighton, L. R. Price, and X. Siemens, Phys. Rev. D 79, 084030 (2009), URL http://link.aps.org/doi/10.1103/PhysRevD.79.084030.

M. E. d. S. Alves and M. Tinto, Phys. Rev. D 83, 123529 (2011), URL http://link.aps.org/doi/10.1103/PhysRevD.83.123529.

R. N. Manchester, G. B. Hobbs, A. Teoh, and M. Hobbs, Astrophys. J. 129, 1993 (2005), URL http://stacks.iop.org/1538-3881/129/i=4/a=1993.

J. M. Cordes and T. J. W. Lazio, ArXiv Astrophysics e-prints (2002), arXiv:astro-ph/0207156. 